\title{
基于多源卫星数据的小型水体蓝藻水华联合监测一一天津于桥水 库为例"
}

\author{
房 旭 ${ }^{1,2}$, 段洪涛 ${ }^{2 * *}$, 曹志刚 ${ }^{2}$, 沈 明 $^{2}$, 葛小三 ${ }^{1}$ \\ ( 1 : 河南理工大学测绘与国土信息工程学院,焦作 454000$)$ \\ (2: 中国科学院南京地理与湖泊研究所,南京 210008)
}

\begin{abstract}
摘 要: 近年来水体富营养化呈扩张趋势, 蓝藻水华不仅在太湖等大型湖泊频发, 水面面积较小的天津于桥水库等也形 势严峻, 亟需加强卫星遥感监测. 但是, 以往在太湖等业务化使用非常成功的 MODIS 等卫星数据 (约 $500 \mathrm{~m}$ ), 由于空间分 辨率较低, 难以满足小型水体的监测要求; 而 Landsat -8 等空间分辨率较高的卫星数据 $(>30 \mathrm{~m})$, 通常重返周期较长, 无法 满足水华高频监测需求. 本文以天津市于桥水库 (面积约 $80 \mathrm{~km}^{2}$ ) 为研究区, 针对常用的卫星数据, 从空间、时间、光谱范 围和数据可获取性共 4 个方面, 评价不同卫星数据蓝藻水华监测能力和算法, 同时对不同卫星监测结果一致性进行评估. 结果表明: (1) 篮选出国产 HJ-1AlB CCD 、GF-1 WFV 和美国 Landsat-8 OLI 这 3 种卫星波段合适, 空间分辨率较高, 适用于 桥水库蓝澡水华监测, 但考虑到其重返周期较长, 建议多星联合观测; (2) 各个卫星监测结果与卫星影像目视解译结果基 本一致,均方根误差和相对误差均分别控制在 $0.78 \mathrm{~km}^{2}$ 和 $4.9 \%$ 以内; (3) 不同卫星监测结果一致性良好,一致性精度达到 $99.5 \%$; (4) 根据历史影像结果, 发现于桥水库 2016 年水质开始呈富营养化, 藻华现象在夏、秋两季最为严重. 研究表明, 针对小型水面水体蓝藻水华监测, 利用较高分辨率数据联合监测, 是一种有效的替代策略, 今后可在更多小型水域推广. 关键词: 水体富营养化; 蓝藻水华; 高分辨率卫星数据;联合监测;于桥水库
\end{abstract}

\section{Remote monitoring of cyanobacterial blooms using multi-source satellite data: A case of Yuqiao Reservoir, Tianjin}

\author{
FANG $\mathrm{Xu}^{1,2}$, DUAN Hongtao ${ }^{2 * *}$, CAO Zhigang ${ }^{2}$, SHEN Ming ${ }^{2}$ \& GE Xiaosan ${ }^{1}$ \\ (1: School of Surveying and Land Information Engineering of Henan Polytechnic University, Jiaozuo 454000, P.R. China) \\ (2: Nanjing Institute of Geography and Limnology, Chinese Academy of Sciences, Nanjing 210008, P.R.China)
}

\begin{abstract}
In recent years, there is a growing eutrophication of surface freshwaters. In many cases, this has resulted in an increase in the occurrence and persistence of cyanobacterial blooms, not only large lakes, but also in small reservoirs. There is an urgent need for new and better monitoring approaches. Satellite based remote sensing is one important and increasing accessible tool. However, the application of MODIS and other satellite data with low spatial resolution (about $500 \mathrm{~m}$ ) but high temporal frequency was limited to larger lakes. While with higher spatial resolution satellite data $(<30 \mathrm{~m})$, such as Landsat- 8 , have longer return periods, making them less useful for blooms monitoring. This study explores the usage of the Chinese HJ-1A \B CCD and GF-1 WFV sensors, together with the United States Landsat-8 OLI and other high-resolution satellite data for joint observation of cyanobacteria blooms in a small reservoir (Yuqiao Reservoir, Tianjin). An algal extraction was developed for each satellite sensor, and the output of different satellite sensors was evaluated. The results indicate that: (1) the combined satellite monitoring provided consistent results when compared to the visual interpretation of multiband satellite images with a mean square root error and relative error of $0.78 \mathrm{~km}^{2}$ and $4.9 \%$; $(2)$ different satellite sensors provided consistent results, with an accuracy of 99.5\% ; (3) According to the research in 2016, the water quality of Yuqiao Reservoir was eutrophic, and algal bloom was the most serious in the two seasons of summer and autumn. The use of combined observations of high-resolution data was an effective, alternative strategy for monitoring cyanobacterial blooms in small
\end{abstract}

* 江苏省杰出青年基金项目 (BK20160049) 和国家自然科学基金项目 (41671358) 联合资助. 2017-09-22 收稿; 2017-11-29 收修改稿. 房旭 (1991 ), 男,硕士研究生; E-mail:303085765@ qq.com.

** 通信作者; E-mail: htduan@ niglas.ac.cn. 
waterbodies, opening up new possibilities to improve monitoring of these important freshwater environments.

Keywords: Water eutrophication; cyanobacterial blooms; high-resolution satellite data; multi-source remote sensing; Yuqiao Reservoir

湖泊和水库是我国主要的水源地,为全国 $85 \%$ 的人口提供饮用水源. 但是, 近几十年来湖泊和水库水质明 显下降, 有 $1 / 3$ 水库达到富营养化水平, 先后发生了蓝藻水华 ${ }^{[1]}$. 比如, 太湖、巢湖、三峡水库、广东汤溪水库和 长春石头口门水库等,都频频暴发水华,危害饮用水安全,造成了不可估量的损失 ${ }^{[2-4]}$. 事实上,2007 年太湖蓝 藻水华引起的水危机事件之后, 蓝藻灾害的整体应对策略已经从“被动应急”转为“主动防控” ${ }^{[5]}$. 主动防控, 就需要及时掌握湖库蓝藻水华状况,在发生危机之前及时采取措施,卫星遥感监测就变得尤为关键.

卫星遥感技术具有监测范围广、周期性重复和成本低等特点, 因此利用卫星遥感技术可以全面快速地 获取蓝藻水华的时空分布, 有效实现动态监测. 目前大型湖泊如太湖、巢湖等蓝藻水华监测, 主要采用 MODIS 数据, 获得广泛应用. 段洪涛等 ${ }^{[6]}$ 基于多源卫星利用单波段和波段比值的方法得到太湖蓝藻水华监 测的普适模式; 李旭文等 ${ }^{[7]}$ 利用归一化植被指数 ( normalized difference vegetation index, $N D V I$ ) 对太湖蓝藻水 华强度进行了划分; Hu 等 ${ }^{[8-9]}$ 利用 MODIS 的瑞利反射率数据 (rayleigh-corrected reflectance, $R_{\mathrm{rc}}$ ) 提出浮游藻 类指数 (floating alage index, $F A I$ ), 并应用于太湖蓝藻水华的监测中;Zhang 等 ${ }^{[10]}$ 针对 MODIS 数据提出了基 于亚像元算法估计蓝藻水华覆盖面积的研究. 事实上, 目前已经形成基于 MODIS 数据的太湖和巢湖等大型 水体蓝藻水华业务化监测方案, 这主要由于其空间分辨率虽然适中 (500 m 左右), 但时间分辨率高, 一天可 以实现 2 次监测, 对于快速变化的蓝藻水华来说, 是相对理想的数据源.

相对于太湖等大型水体, 水域面积较小的水体, MODIS 数据空间分辨率显然无法满足要求; 但是, 我国 的高分 1 号卫星 ( GF-1 WFV)、环境卫星 ( HJ-1A \B CCD) 以及美国的陆地卫星 (Landsat-8 OLI) 等空间分辨率 优于 $30 \mathrm{~m}$, 可有效提高小型水体蓝藻水华的监测精度. 事实上, 由于蓝藻水华变化快, 卫星监测既要能看得 见藻华, 也要高频监测, 特别是业务管理部门希望最好每天都有数据. 但是, 这些高分辨率卫星数据, 一般重 返周期比较长, 短则 $2 \sim 3$ 天, 长则半个月, 再加上云雨等气象因素影响, 使得单个传感器监测无法满足水华 高频监测要求 ${ }^{[11]}$. 因此, 采用多个高空间分辨率卫星传感器进行蓝藻水华联合监测, 就成为必然的选择.

在实际使用中, 由于不同卫星传感器数据性能和波段等不一致, 必然导致蓝藻水华监测的一致性问题; 特别是空间分辨率不同引起的空间尺度效应. 本文选择小型水体一一天津于桥水库, 利用 HJ-1A B B GF-1 和 Landsat-8 等高分辨率卫星, 尝试对其蓝藻水华进行日常监测, 主要目标: (1)评估不同卫星的蓝藻水华监测能 力; (2)研制不同卫星的水华监测算法, 评估其水华产品一致性; (3)分析于桥水库 2016 年长时间序列蓝藻水 华时空分布规律. 总体上, 本文一方面提出了适合小型水体的蓝藻水华卫星监测方案, 有助于更多小型水体 得到有效监测; 另一方面, 对于桥水库蓝藻水华发生历史进行追溯, 有利于当地管理部门更好治理蓝藻水华 等水环境问题.

\section{1 研究区域与数据}

\section{1 研究区概况}

于桥水库 $\left(39^{\circ} 99^{\prime} 71^{\prime \prime} \sim 40^{\circ} 07^{\prime} 44^{\prime \prime} \mathrm{N}, 117^{\circ} 43^{\prime} 09^{\prime \prime} \sim 117^{\circ} 68^{\prime} 26^{\prime \prime} \mathrm{E}\right.$ ) 位于天津市蓟县城东, 最大回水东西长约 30 $\mathrm{km}$, 南北宽 $8 \mathrm{~km}$, 最大淹没面积 $250 \mathrm{~km}^{2}$ (正常蓄水位时淹没面积 $\left.86.8 \mathrm{~km}^{2}\right)^{[12]}$, 总库容 15.59 亿 $\mathrm{m}^{3}, 1983$ 年成 为 “引㴒人津输水工程” 中重要的调蓄水库, 是天津市主要饮用水源地之一 ${ }^{[13]}$ (图 1). 于桥水库控制流域面积 $2060 \mathrm{~km}^{2}$, 占州河总流域面积的 96\% ; 州河由沙河、淋河和黎河三大支流汇合而成即人库河流, 各支流上游沟涧 甚多, 支流分散成辐射状汇集于州河盆地, 水库库区即位于该盆地. 近年来, 由于上游潘家口和大黑汀水库水 质严重恶化, 加上流域内人库河流污染物的汇人, 致使大量营养盐在于桥水库积累 ${ }^{[14]}$, 超出水库水体的自净能 力, 破坏水库的生态平衡, 造成于桥水库藻类水华频繁暴发 ${ }^{[15]}$, 直接威胁天津市的供水安全.

\section{2 遥感数据}

卫星遥感具有监测范围大、资料获取方便和动态监测等特点, 因此越来越受到人们的重视. 本文选择了 可获取的 8 种国内外卫星数据用于于桥水库蓝藻水华的监测和评价, 具体参数见表 1 . 另外, 选取了于桥水 


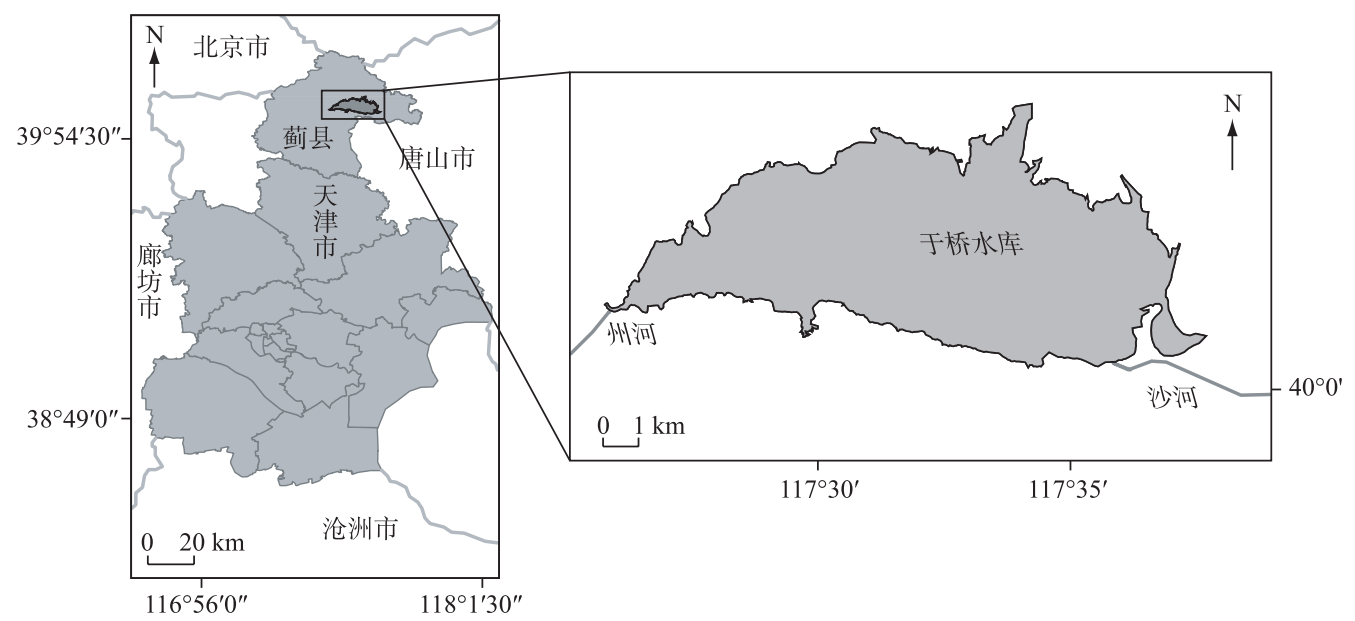

图 1 研究区域示意图

Fig. 1 Sketch of the study area

库历年卫星影像, 发现 2016 年于桥水库开始大规模暴发蓝藻水华, 因此, 本研究集中于 2016 年的卫星数据.

表 1 主要卫星参数

Tab. 1 Satellite parameter list

\begin{tabular}{ccccccc}
\hline 卫星 & 传感器 & 空间分辨率/m & 重返周期/d & 波段数 & 光谱范围/nm & 数据可获取性 \\
\hline TERRA/AQUA & MODIS & $250 / 500 / 1000$ & $1 \sim 2$ & 36 & $412 \sim 1050$ & 免费下载 \\
GF-4 & PMI & 50 & $20 \mathrm{~s}$ & 5 & $450 \sim 900$ & 免费下载 \\
Landsat-8 & OLI & $15 / 30$ & $16 \sim 18$ & 7 & $430 \sim 1380$ & 免费下载 \\
HJ-1A $\backslash B$ & CCD & 30 & 4 & 4 & 蓝、绿、红、近红外 & 免费下载 \\
CBERS-04 & MUX & 20 & 26 & 4 & $450 \sim 890$ & 免费下载 \\
GF-1 & WFV & 16 & 4 & 4 & 蓝、绿、红、近红外 & 免费下载 \\
ZY-3 & MUX & 6 & 5 & 4 & $450 \sim 890$ & 付费下载 \\
ZY-1 02C & MUX & $5 / 10$ & 3 & 4 & $510 \sim 890$ & 付费下载 \\
\hline
\end{tabular}

1.2.1卫星数据篮选标准 蓝藻水华变化快, 数据需求急, 对时间和空间分辨率的要求都很高. 本文对当前 可用的主要 8 种卫星, 根据其空间分辨率、时间分辨率、波段设置以及数据可获取性 4 方面, 对数据进行评价 和笁选,用于于桥水库蓝藻水华监测.

1.2.2 卫星遥感数据遴选 目前最为常用的 MODIS 数据, 虽然具有较高的时间分辨率和光谱分辨率, 被广泛 应用于大型内陆湖泊的蓝藻水华监测 ${ }^{[16]}$; 但是其空间分辨率低 $(250 、 500$ 和 $1000 \mathrm{~m}$ ), 在小型水域的识别能 力上, 藻华提取精度存在明显的误差 (图 2a). 高分四号卫星 (GF-4) 是地球同步轨道卫星,可实时观测研究 区动态变化, 较多的波段设置能满足蓝藻水华动态监测的需求, 但是 $50 \mathrm{~m}$ 的空间分辨率不能满足于桥水库 的水华识别 (图 2b). Landsat- 8 拥有 $30 \mathrm{~m}$ 的空间分辨率, 可以清楚看到蓝藻水华; 但其 $16 \mathrm{~d}$ 的重访周期, 限 制了对于桥水库水华的动态监测能力 (图 2c). 这也说明, $80 \mathrm{~km}^{2}$ 左右大小的于桥水库, 至少需要 $30 \mathrm{~m}$ 以上 的空间分辨率. 高于 $30 \mathrm{~m}$ 空间分辨率的卫星还有 5 种,都拥有近红外和可见光波段,可用于水华监测 (表 1); 但是其重返周期普遍在 3 天以上; 另外, 分辨率在 $10 \mathrm{~m}$ 以上的 ZY-3、ZY-1 02C MUX 数据都需要另外付 费购买,数据获取不方便. 因此, 综合考虑空间、时间、光谱范围和数据可获取性 4 个因素 (表 2), 可以用于 于桥水库水华监测的主要卫星有 Landsat-8、HJ-1A $\backslash \mathrm{B}$ 和 GF-13 种; 但其重返周期都无法实现每天覆盖, 满足 业务管理部门要求的蓝藻水华高频观测需求, 因此本文决定联合使用这 3 颗卫星用于蓝藻水华遥感监测, 弥补时间分辨率不足,共选取 2016 年无云或少云影像 66 景(表 3). 

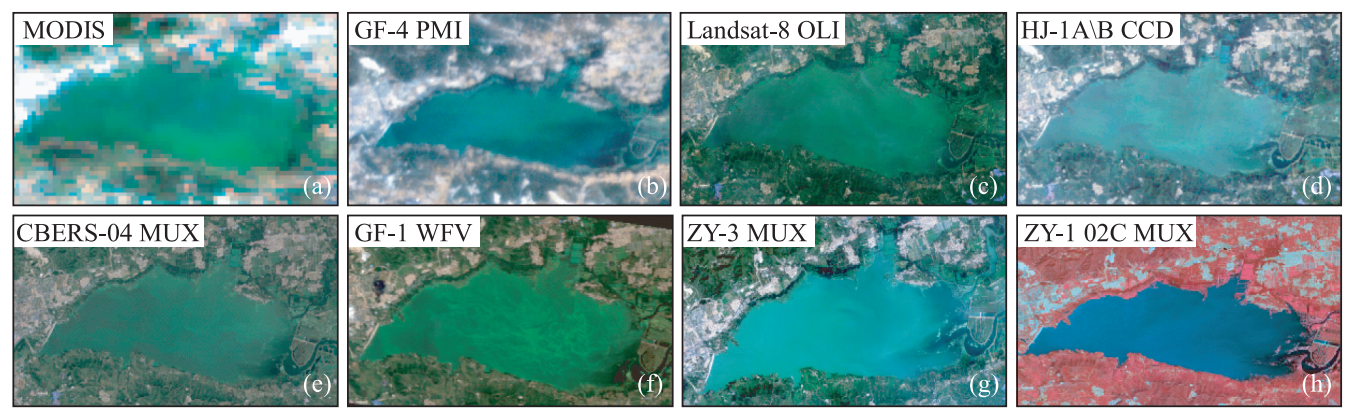

图 2 卫星真彩色合成图对比:MODIS (a)、GF-4 PMI (b)、Landsat-8 OLI (c)、HJ-1A \B CCD (d)、CBERS-04 MUX (e)、GF-1 WFV (f)、ZY-3 MUX (g) 和 ZY-1 02C MUX (h) (ZY-1 02C MUX 只有假彩色合成波段)

Fig.2 True color synthetic maps of different satellite sensors

表 2 卫星遥感数据篮选

Tab.2 Satellite remote sensing data filtering table

\begin{tabular}{cccccc}
\hline 卫星 & 传感器 & 空间分辨率/ $\mathrm{m}$ & 重返周期/d & 光谱范围/ $\mathrm{nm}$ & 数据可获取性 \\
\hline TERRA/AQUA & MODIS & & $\sqrt{ }$ & $\sqrt{ }$ & $\sqrt{ }$ \\
GF-4 & PMI & & $\sqrt{ }$ & $\sqrt{ }$ & $\sqrt{ }$ \\
Landsat-8 & OLI & $\sqrt{ }$ & $\sqrt{ }$ & $\sqrt{ }$ \\
HJ-1A $\backslash B$ & CCD & $\sqrt{ }$ & $\sqrt{ }$ & $\sqrt{ }$ \\
CBERS-04 & MUX & $\sqrt{ }$ & $\sqrt{ }$ & $\sqrt{ }$ \\
GF-1 & WFV & $\sqrt{ }$ & $\sqrt{ }$ & $\sqrt{ }$ \\
ZY-3 & MUX & $\sqrt{ }$ & $\sqrt{ }$ & $\sqrt{ }$ \\
ZY-1 02C & MUX & $\sqrt{ }$ & $\sqrt{ }$ & & \\
\hline
\end{tabular}

表 32016 年卫星影像获取时间

Tab.3 2016 satellite imagery acquisition time

\begin{tabular}{|c|c|c|}
\hline 卫星 & 传感器 & 时间(月-日) \\
\hline $\mathrm{HJ}-1 \mathrm{~A} \backslash \mathrm{B}$ & CCD & $\begin{array}{l}02-29,03-20,03-25,04-03,04-18,05-04,05-18,05-22,05-26,06-16,07-06,07-27, \\
08-08,08-21,09-09,09-19,10-01,10-17,11-08,11-15,11-24,11-28,12-09,12-10, \\
12-14,12-15\end{array}$ \\
\hline GF-1 & WFV & $\begin{array}{l}02-24,03-11,03-23,04-01,04-09,04-25,04-30,05-08,05-28,05-29,06-05,06-22, \\
07-05,08-22,08-26,08-27,09-08,09-19,09-20,09-28,09-29,10-03,10-14,10-23, \\
10-28,11-16,12-16\end{array}$ \\
\hline Landsat- 8 & OLI & $\begin{array}{l}02-23,03-10,04-11,04-18,05-04,05-13,05-29,07-16,08-08,09-09,09-18,10-11, \\
12-14\end{array}$ \\
\hline
\end{tabular}

\section{2 研究方法}

\section{1 蓝藻水华提取方法}

首先对相关影像进行辐射定标和几何纠正, 选择经过实测地理数据坐标校正过的, 无云且成像质量好 的一景 Landsat-8 OLI 影像为基准影像, 通过 ENVI 5.1 对 HJ-1A $\backslash \mathrm{B}$ 和 GF-1 影像进行几何纠正, 误差小于 0.5 个像元, 然后采用 FLAASH 大气校正得到图像反射率; 并在 ERDAS 中利用于桥水库矢量边界, 通过掩模技 术提取于桥水域, 去除于桥水库周边及其植被. 具体流程为 (图 3):(1) 将多源高分辨数据进行遥感图像预 处理, 再利用处理后的影像计算 $N D V I$ 指数, 并统计藻华和非藻华的 $N D V I$ 阈值; (2) 利用藻华和非藻华阈值 
参与像元生长算法迭代运算, 当连续 2 次计算的绝对误差或者相对误差达到一个最小值时, 循环结束; (3) 利用尺度转换重采样对计算结果进行转换,最后输出面积分布图.

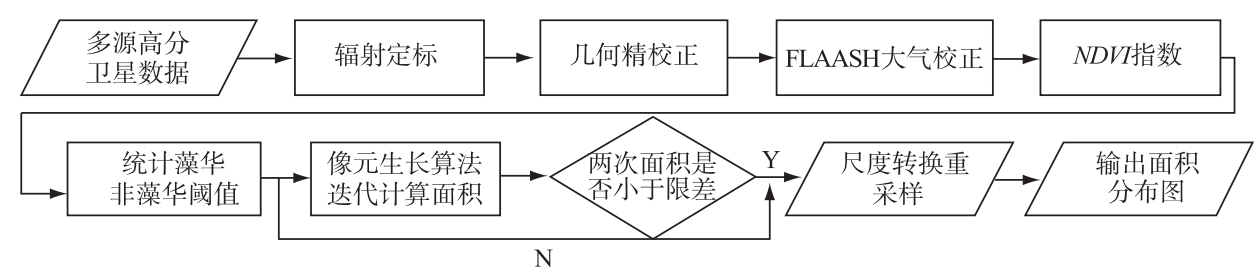

图 3 算法流程图

Fig.3 Algorithm flow chart

综合考虑 3 种卫星数据波段设置, $N D V I$ 指数对植被敏感, 以及蓝藻水华在近红外波段具有类似于植被 光谱曲线特征的 “陡坡效应” ${ }^{[17]}$. 本研究中对大气校正的绝对准确度的要求并不重要 ${ }^{[18]}$, 因为使用红和近 红外波段可以有效减少使用蓝绿波段时大气的影响和有色溶解性有机物 (CDOM) 的干扰问题 ${ }^{[19]}$, 因此选择 $N D V I$ 指数作为监测蓝藻水华暴发情况的基本指数：

$$
N D V I=\frac{\rho_{\text {nir }}-\rho_{\text {red }}}{\rho_{\text {nir }}+\rho_{\text {red }}}
$$

式中, $\rho_{\text {nir }}$ 和 $\rho_{\text {red }}$ 分别代表近红外波段和红光波段的反射率.

由于藻华区域与水体光谱特征通常存在较大差别, 在蓝藻水华和非水华的边界区域, $N D V I$ 结果会有较 大的差异, 像元变化率也较大 ${ }^{[20]}$; 因此通过计算 $N D V I$ 卫星产品的像元坡度, 统计坡度最大处的 $N D V I$ 值, 并 利用均值减去 2 倍标准差, 即可确定水华阈值. 实际上该方法已经在太湖等区域成功实践 ${ }^{[19]}$. 本文以 $\mathrm{HJ}-1 \mathrm{~A} \backslash \mathrm{B}$ CCD 卫星影像为例, 依据坡度与统计分析的方法求出每幅影像的阈值, 作出统计直方图, 得出单景 2016 年 9 月 12 日影像藻华阈值为 0.085 (图 4a) ; 然后对 HJ-1A \B 统计的 26 景影像國值进行平均处理, 最 终得到区分蓝藻水华与清洁水体的统一阈值为 -0.064 (图 4b), 该阈值即为算法中纯蓝藻水华像元的输人阈 值. 同理, GF-1 藻华的统一阈值为 -0.083 (图 4d) ; Landsat-8 由于影像数量限制, 无法确定统一阈值, 但在实 际使用中为保证精度, 每一景单独确定阈值.

此外,采用等间隔方法确定藻华聚集强度分级阈值,将于桥水库蓝藻水华区分成低强度聚集区和高强 度聚集区, 确定水华强度分布可以为蓝藻水华高强度聚集区的重点治理提供参考.

\section{2 像元生长算法统计面积}

由于于桥水库水域面积较小, 虽利用环境卫星藻华平均阈值 $N D V I_{\text {algae }}=-0.064$ 提取出了高浓度蓝藻水 华 (以 $\mathrm{HJ}$ 卫星为例), 但依然存在着许多水和蓝藻混合的像元, 利用像元生长算法可有效提高统计蓝藻水华 的面积精度. 像元生长算法 (algae pixel-growing algorithm, APA) 是通过判定卫星影像中的藻华 “生长点像 元”, 采用临近像元相关和逐渐扩展的思路, 从而精确计算出混合像元的藻华盖度, 这里所说的盖度是指某 一混合像元中藻华完全覆盖的面积占该像元面积的百分比;具体计算过程见参考文献 [19].

\section{3 不同影像水华提取结果尺度转换}

尺度转换是指将某尺度上所获取的知识和信息扩展到其他尺度上的过程 ${ }^{[21]}$; 事实上, 即使是相同时相 的相同地物, 由于尺度效应, 不同卫星提取的水华信息也不尽相同. 本研究联合 HJ-1A \B CCD、GF-1 WFV 和 Landsat-8 OLI 3 个不同空间分辨率的卫星传感器监测蓝藻水华, 通过重采样将不同卫星空间分辨率转换到 同一标准.

选择双线性内插法进行重采样,该方法具有平均化的滤波效果, 图像边缘受到平滑作用, 而产生一个连 贯的输出影像, 辐射保真度较好. 根据双线性内插法, 将 $N D V I$ 结果重采样至 $30 \mathrm{~m}$, 重新利用算法计算藻华盖 度, 当循环满足条件时,即输出经重采样转换后的藻华分布图.

采用均方根误差 (root mean square error, RMSE) 和相对误差 (relative error, $R E$ ) 对 APA 算法得到的结果 精度进行评判: 

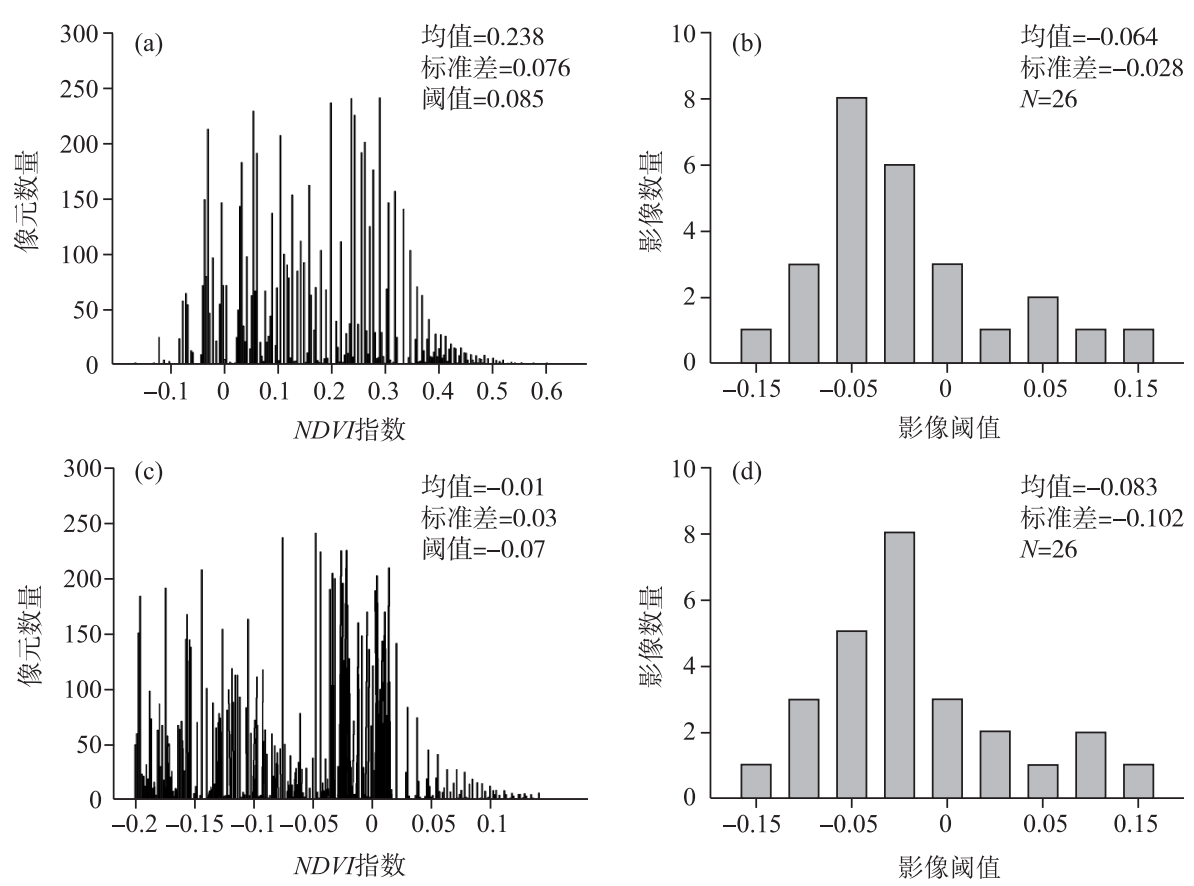

图 4 2016-9-12 HJ-1A \B 和 2016-8-27 GF-1 卫星 $N D V I$ 蓝藻水华分界数据直方图 (a,c)、 $N D V I$ 阈值统计直方图 $(\mathrm{b}, \mathrm{d})$

Fig.4 2016-9-12 HJ-1A \B and 2016-8-27 GF-1 satellite NDVI watershed data histogram(a, c), NDVI threshold statistic histogram $(\mathrm{b}, \mathrm{d})$

$$
\begin{gathered}
R M S E=\sqrt{\frac{\sum_{i=1}^{n}\left(d_{p, i}-d_{m, i}\right)^{2}}{n}} \\
R E=\frac{1}{n} \sum_{i=1}^{n} \frac{\left|d_{p, i}-d_{m, i}\right|}{d_{m, i}} \times 100 \%
\end{gathered}
$$

式中, $d_{\mathrm{p}, i}$ 和 $d_{\mathrm{m}, i}$ 分别代表第 $i$ 个模拟值和实测值, $n$ 为样点总数.

\section{3 结果与分析}

\section{1 于桥水库蓝藻水华提取结果评价}

3.1.1 不同影像提取结果 为了验证 APA 算法在不同卫星传感器的提取结果, 选取 HJ-1A \B CCD 、 Landsat-8 OLI 和 GF-1 WFV 提取结果与原影像假彩色合成图进行对比 (图 5). 可以看出, APA 算法在不同卫星传感器 上得到的藻华面积分布图与原始影像中,藻华空间分布情况基本一致.

由于个别影像的蓝藻水华阈值与统一的蓝藻水华阈值有较大差异, 为了更好地对提取结果进行验证, 随机选取 6 组 HJ-1A \B CCD、GF-1 WFV 影像, 采用坡度分析与目视解译相结合方法确定出 CCD、WFV 单景 影像藻华阈值和清洁水体阈值,然后根据单景的阈值确定出藻华面积对 APA 算法提取的结果进行验证, 6 组单景阈值的藻华面积介于 $4.48 \sim 22.83 \mathrm{~km}^{2}$ 之间. APA 算法得到的结果与 CCD、WFV 影像单景阈值提取的 结果较为接近, 根据公式 (2)、(3) 分别得到 $R M S E$ 为 $0.78 、 0.75 \mathrm{~km}^{2}, R E$ 分别为 $4.6 \% 、 4.9 \%$ (图 6). 本文所提 出的算法相较于传统的目视解译的方法, 不但降低了人为因素的干扰, 而且统一的阈值提升了业务效率, 更 能够精确提取出混合像元中的蓝藻水华, 藻华面积监测结果更加精确. 另外, 由于 Ladnsat-8 OLI 影像数量较 少,本文不做分析. 

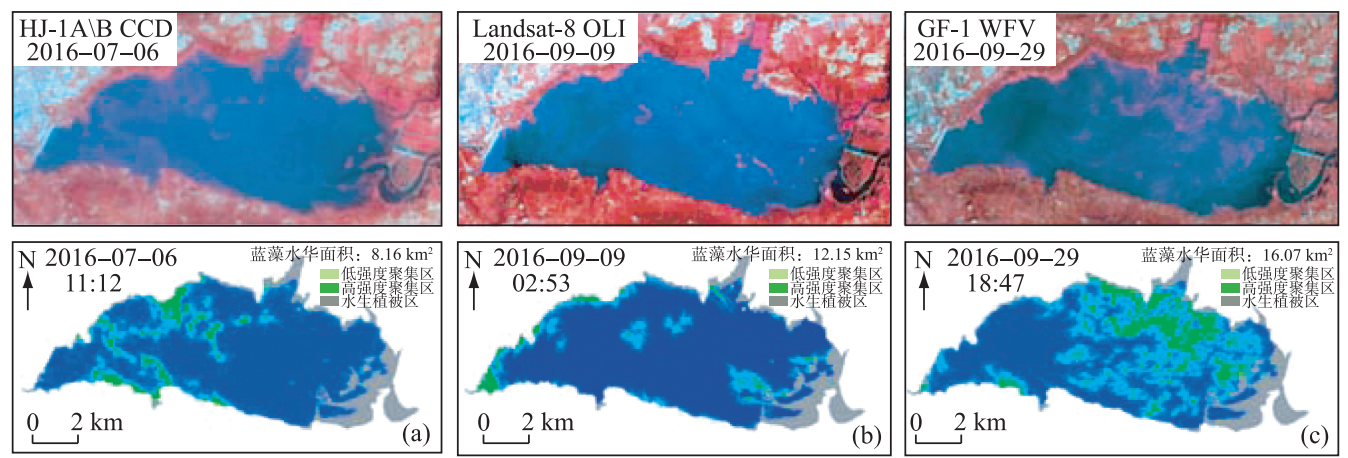

图 5 APA 算法与假彩色影像对比图:HJ-1A \B CCD(a)、Landsat-8 OLI (b) 和 GF-1 WFV(c)

Fig.5 Comparison between APA algorithm and false color image:

HJ-1A $\backslash$ B CCD (a), Landsat-8 OLI (b) and GF-1 WFV (c)

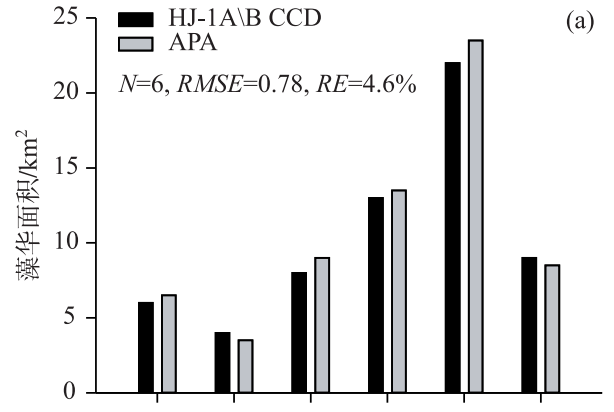

6组HJ-1 A $\backslash B$ CCD影像和APA影像

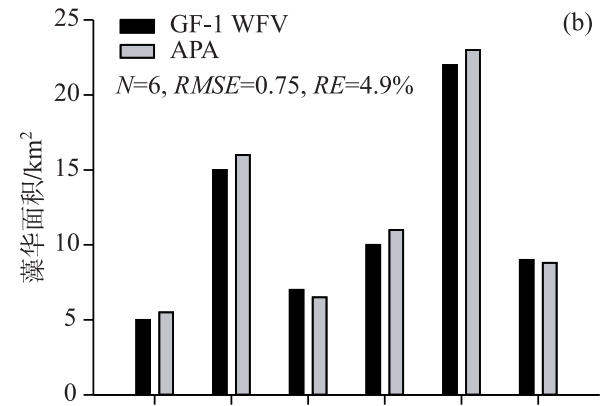

6组GF-1 WFV 影像和APA影像

图 6 随机 6 组单景阈值提取结果与 APA 算法提取结果对比: HJ-1A \B CCD (a) 和 GF-1 WFV (b)

Fig.6 Contrast with the results obtained from the visual interpretation of random 6 groups and the APA algorithm(HJ-1A $\backslash B$ CCD (a) and GF-1 WFV (b))

3.1.2 不同影像提取结果对比 选择 HJ-1A $\backslash$ B 影像提取的藻华结果为基准, 对 Landsat-8、GF-1 提取的藻华结果 进行验证. 图 7 分别是 Landsat- $8 、 G F-1$ 和 HJ-1A $\backslash B$ 同一天影像 APA 算法藻华面积结果与假彩色合成图的对 比, 可以看出像元生长算法得到的藻华面积分布图与原影像中藻华空间分布情况一致, 纹理清晰, 表明像元生 长算法计算的藻华面积与实际情况相符, 结果准确. 同样采用验证藻华提取结果的方法, 假设 HJ-1A $\backslash \mathrm{B}$ 卫星提 取蓝藻水华面积为真值, 验证 GF-1、Landsat- 8 卫星提取精度, 得出 Landsat- 8 卫星与 HJ-1 AB 卫星藻华面积均 方根误差 $R M S E$ 为 $0.03 \mathrm{~km}^{2}, R E$ 为 $0.1 \%$; GF-1 卫星与 $\mathrm{HJ}-1 \mathrm{~A} \backslash \mathrm{B}$ 卫星藻华面积均方根误差 $R M S E$ 为 $0.12 \mathrm{~km}^{2}$, $R E$ 为 $0.5 \%$. 但是从空间分布来看, Landsat- 8 卫星和 $\mathrm{HJ}-1 \mathrm{~A} \backslash \mathrm{B}$ 较为相似, 而 GF-1 卫星和 $\mathrm{HJ}-1 \mathrm{~A} \backslash \mathrm{B}$ 差别较大, 前 者的时间分别为当日 $02: 18$ 和 02:53 时间跨度小, 藻华空间分布不会有较大影响; 而后者的时间分别为当日 $11: 13$ 和 15:04 时间跨度较大,再加上风力和日照等因素的影响,藻华空间分布有较大的差异.

3.1.3 验证尺度转换的一致性 不同卫星不同传感器之间存在一定的误差, 得到的藻华结果势必不尽相同. 同样以数据较多的环境卫星数据为基准, 将 2016 年 9 月 9 日 Landsat- 8 影像提取藻华结果和 2016 年 9 月 19 日 GF-1 影像提取藻华结果, 经过重采样尺度转换后, 再次计算基准影像提取结果和校正后的影像结果. 其 中 2016 年 9 月 09 日基准影像 HJ-1A-B 水华面积为 $12.18 \mathrm{~km}^{2}$, Landsat-8 转换前原始提取水华面积为 $12.15 \mathrm{~km}^{2}$, 转换后为 $12.17 \mathrm{~km}^{2}$; 2016 年 9 月 19 日基准影像 $\mathrm{HJ}-1 \mathrm{~A} / \mathrm{B}$ 水华面积为 $21.54 \mathrm{~km}^{2}, \mathrm{GF}-1$ 转换前原 始提取水华面积为 $21.42 \mathrm{~km}^{2}$, 转换后为 $21.52 \mathrm{~km}^{2}$. 综上所述, 2 种卫星 $R E$ 均小于 $0.1 \%$, 且转换卫星藻华空 间分布情况与基准卫星藻华分布情况基本一致, 通过纹理的局部放大图可以看到, 校正后的影像结果更为 

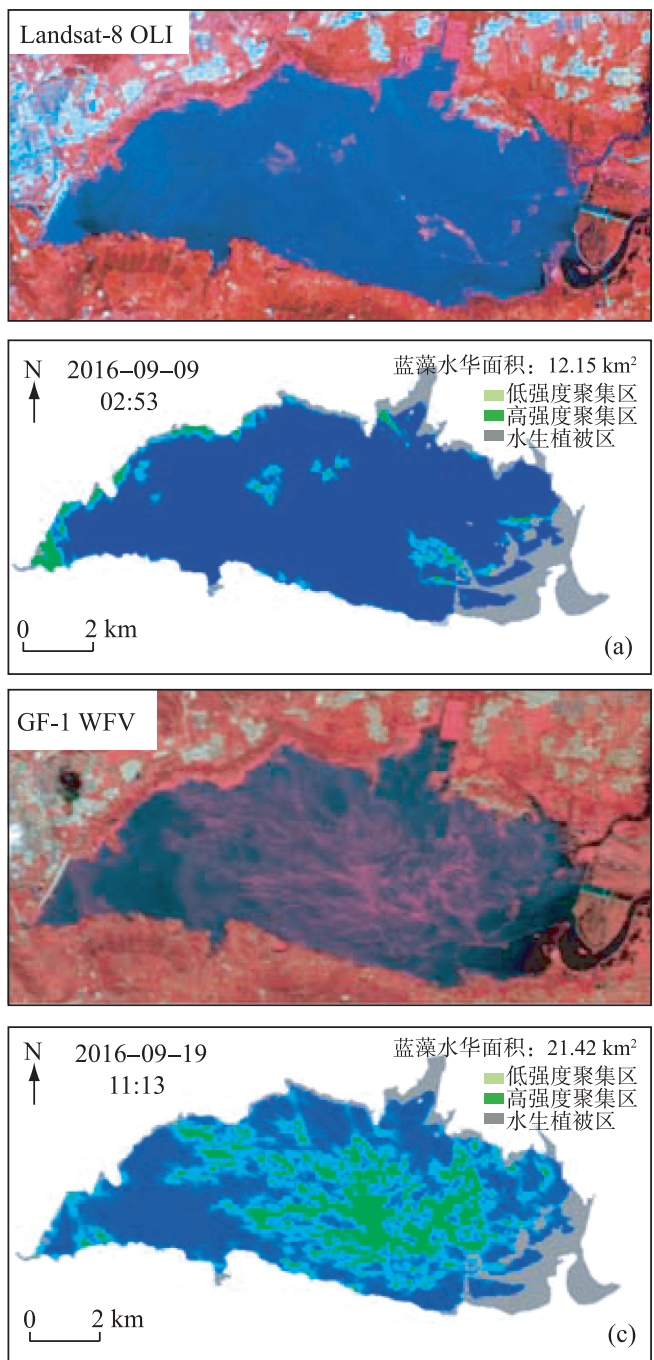
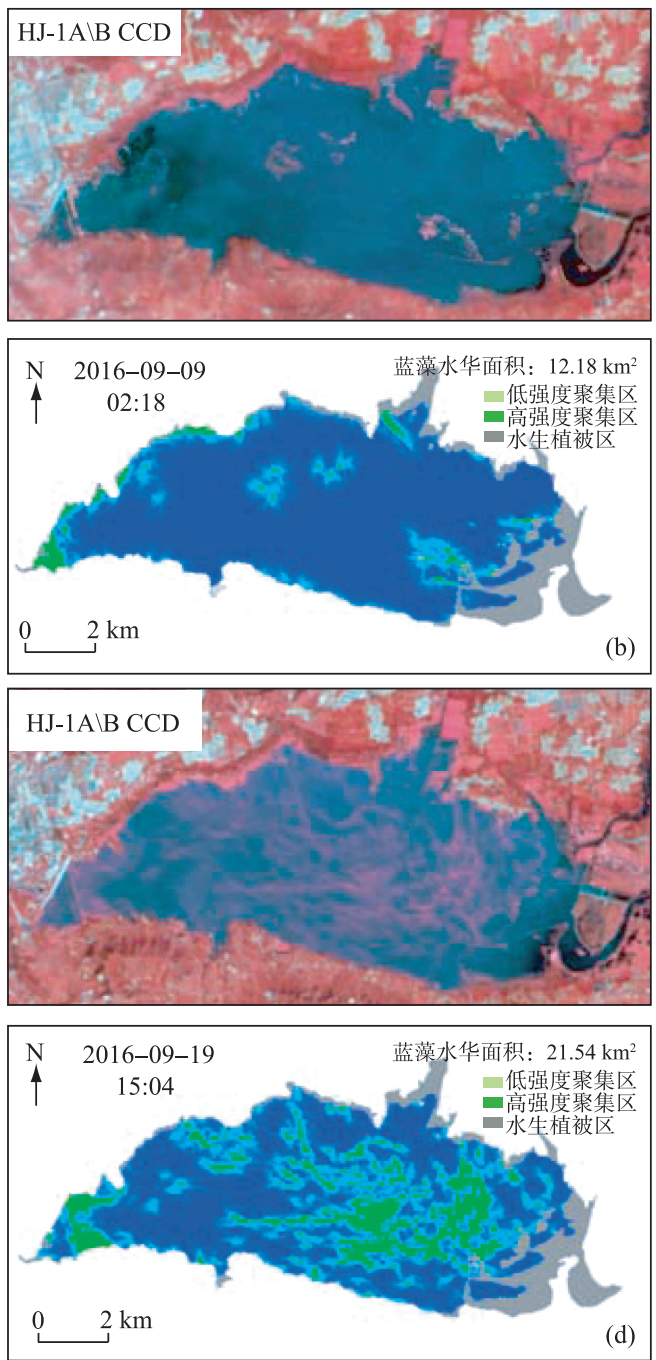

图 7 Landsat-8(a)、HJ-1A $\backslash \mathrm{B}(\mathrm{b})$ 和 GF-1(c)、HJ-1A $\backslash \mathrm{B}(\mathrm{d})$ 藻华面积对比

Fig.7 Landsat-8 (a), HJ-1A $\backslash \mathrm{B}$ (b) and GF-1(c), HJ-1A $\backslash \mathrm{B}(\mathrm{d})$ area comparison of algal blooms

准确(图 8、图 9 分别是 Landsat 卫星、GF-1 卫星转换后的藻华面积空间分布情况).

此外,将 Landsat-8、GF-1 卫星都转换到 HJ-1A \B 卫星基准, 对 Landsat-8、GF-1 藻华盖度结果进行比较 (图 10), 可以看出两者曲线趋势基本一致;但当盖度小于 $20 \%$ 时,卫星之间产生的误差较大,这可能是混合 像元的影响. 其中, 灰色曲线为 HJ-1A $\backslash \mathrm{B}$ 藻华盖度结果, 蓝色曲线为重采样后 Landsat-8、GF-1 藻华盖度结果.

分别统计对应位置 Landsat-8 和 GF-1 影像的 $N D V I$ 和藻华盖度, 作为自变量, 并计算 HJ-1A $\backslash \mathrm{B}$ 影像对应 位置 $N D V$ 和藻华盖度作为因变量. 图 11 为 2016 年 9 月 9 日 Landsat- 8 卫星 $N D V I$ 、藻华盖度与 HJ-1A \B 卫 星相对应的指数的线性关系和 2016 年 9 月 19 日 GF-1 卫星 $N D V I$ 、藻华盖度与 HJ-1A \B 卫星相对应的指数 的线性关系. 通过对比结果发现, HJ-1A $\backslash \mathrm{B}$ 与 Landsat-8 和 GF-1 NDVI 的相关性都很低, $R^{2}$ 分别为 0.27 和 0.28 , 这是由于不同空间分辨率影像所获取的 $N D V I$ 是受非线性的计算方法和地物的空间异质性影响 ${ }^{[11]}$; 而不同卫星藻华盖度之间的相关性很好, $R^{2}$ 分别为 0.83 和 0.56 . 通过对此套算法结果的验证和精度分析,说 明此套算法精度较高, 可用于于桥水库多源卫星联合监测和长时间序列动态监测藻华面积服务. 

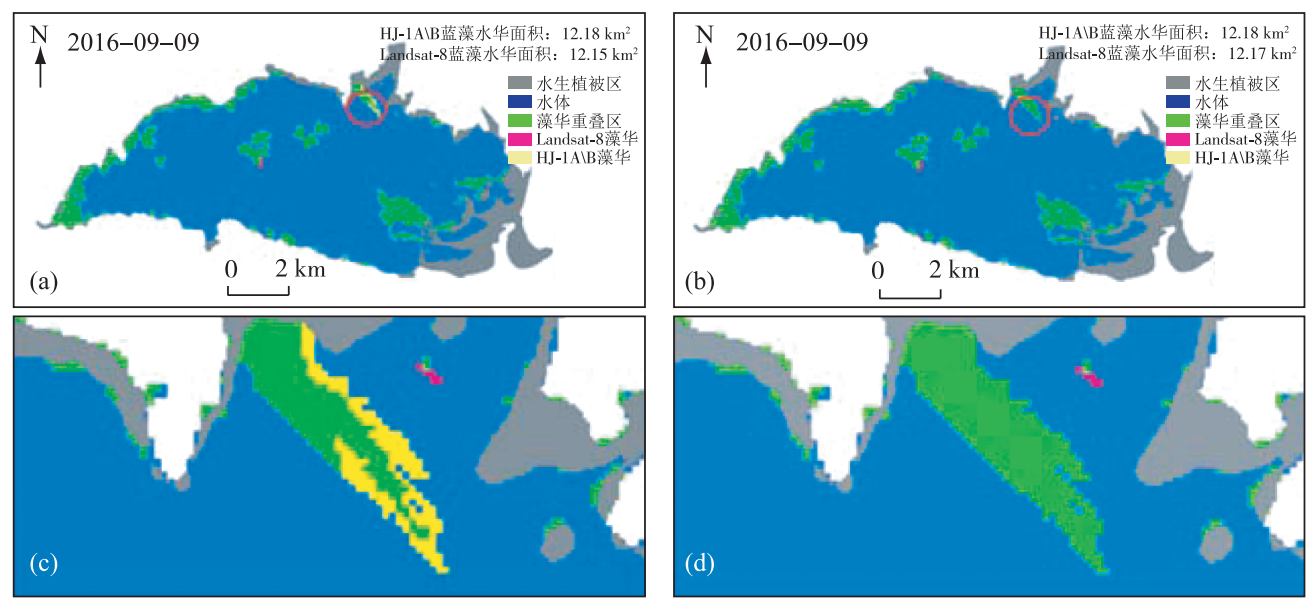

图 8 HJ-1A \B、Landsat-8 卫星原始面积 (a) ; HJ-1A \B、Landsat-8 卫星尺度转换计算面积(b) (图 c、d 分别是图 $\mathrm{a} 、 \mathrm{~b}$ 中红色圆圈放大显示区域)

Fig. 8 HJ-1A $\backslash B$, Landsat-8 satellite original area (a), $\mathrm{HJ}-1 \mathrm{~A} \backslash \mathrm{B}$, Landsat-8 satellite regression model calculating area (b)
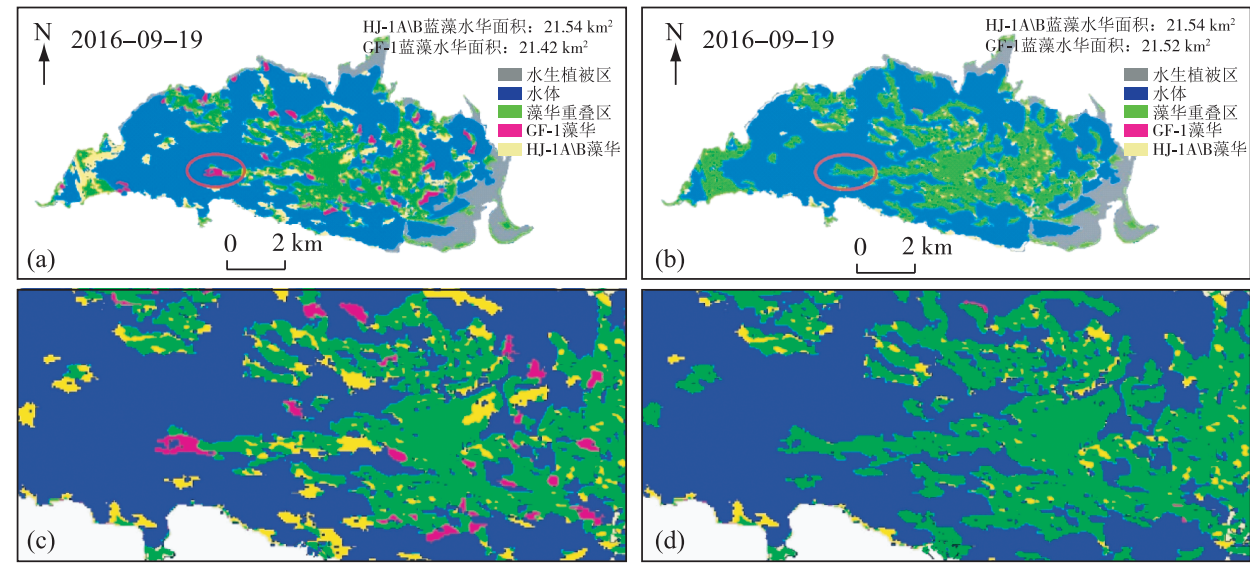

图 9 HJ-1A $\backslash B 、 G F-1$ 卫星原始面积( a ) ; HJ-1A \B、GF-1 卫星尺度转换计算面积 (b)

（图 c、d 分别是图 $\mathrm{a} 、 \mathrm{~b}$ 中红色圆圈放大显示区域）

Fig.9 HJ-1A $\backslash$ B, GF-1 satellite original area (a) ; HJ-1A $\backslash B, G F-1$ satellite regression model calculating area (b)

\section{2 于桥水库蓝藻水华时空分布}

根据统计于桥水库 2016 年蓝藻水华情况, 水库大部分水域都暴发过蓝藻水华,且蓝藻水华最大面积暴发 发生在秋季. 从蓝藻水华分布的空间可以看出,库北和库南发生水华频次最高,严重时涉及整个水库. 其中库 北受人库水源, 上游的潘家口和大黑汀水库源水水质恶化的影响,库南和库东受人类活动的影响, 此外水体表 层的藻类因风浪影响, 藻类和污染物容易堆积, 导致水质恶化, 水体富营养化加剧导致居民用水无法饮用.

选取 2016 年年内遥感卫星监测到的较大型蓝藻水华进行对比, $5 、 6$ 月水库藻华暴发面积较小, 水体状况 较好(图 12). 监测 2016 年一整年的影像数据得到, 冬季藻华暴发面积最小, 春季藻华暴发面积开始增大, 一直 到秋季 9、10 月达到藻华暴发面积的最大值. 这是由于冬季低温等环境因素使得水华蓝藻进人休眠, 并且于桥 水库在 12 月到次年的 3 月完全结冰, 春季受光照和温度等有利因素的影响, 蓝藻得以复苏, 生物量逐渐增加, 夏季温度过高, 蓝藻潜人水面以下, 在秋季更加适宜的水文气象条件下, 藻华暴发情况达到最严重状态. 

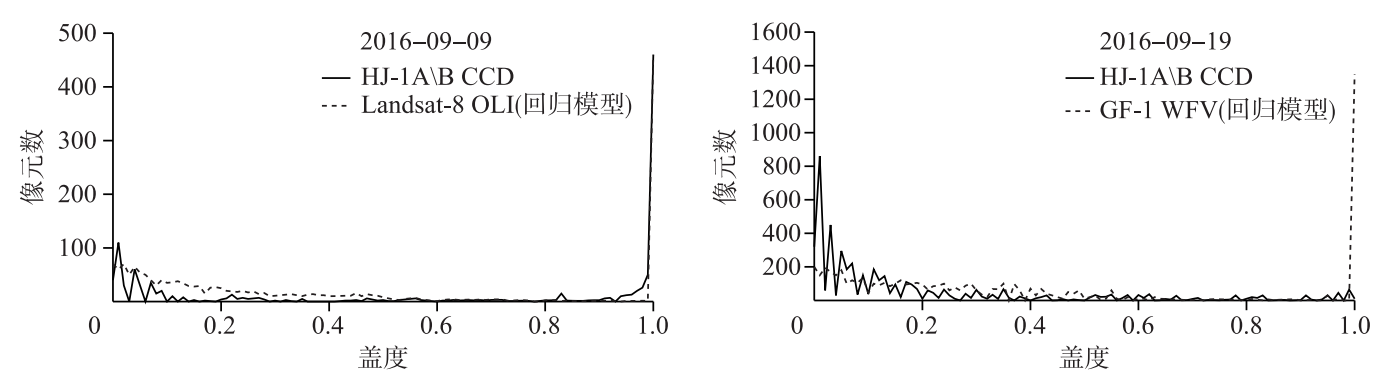

图 10 Landsat-8 OLI、GF-1 WFV 经尺度转换计算藻华盖度

Fig. 10 Landsat-8 OLI and GF-1 WFV by regression model calculation of algal canopy degree plot
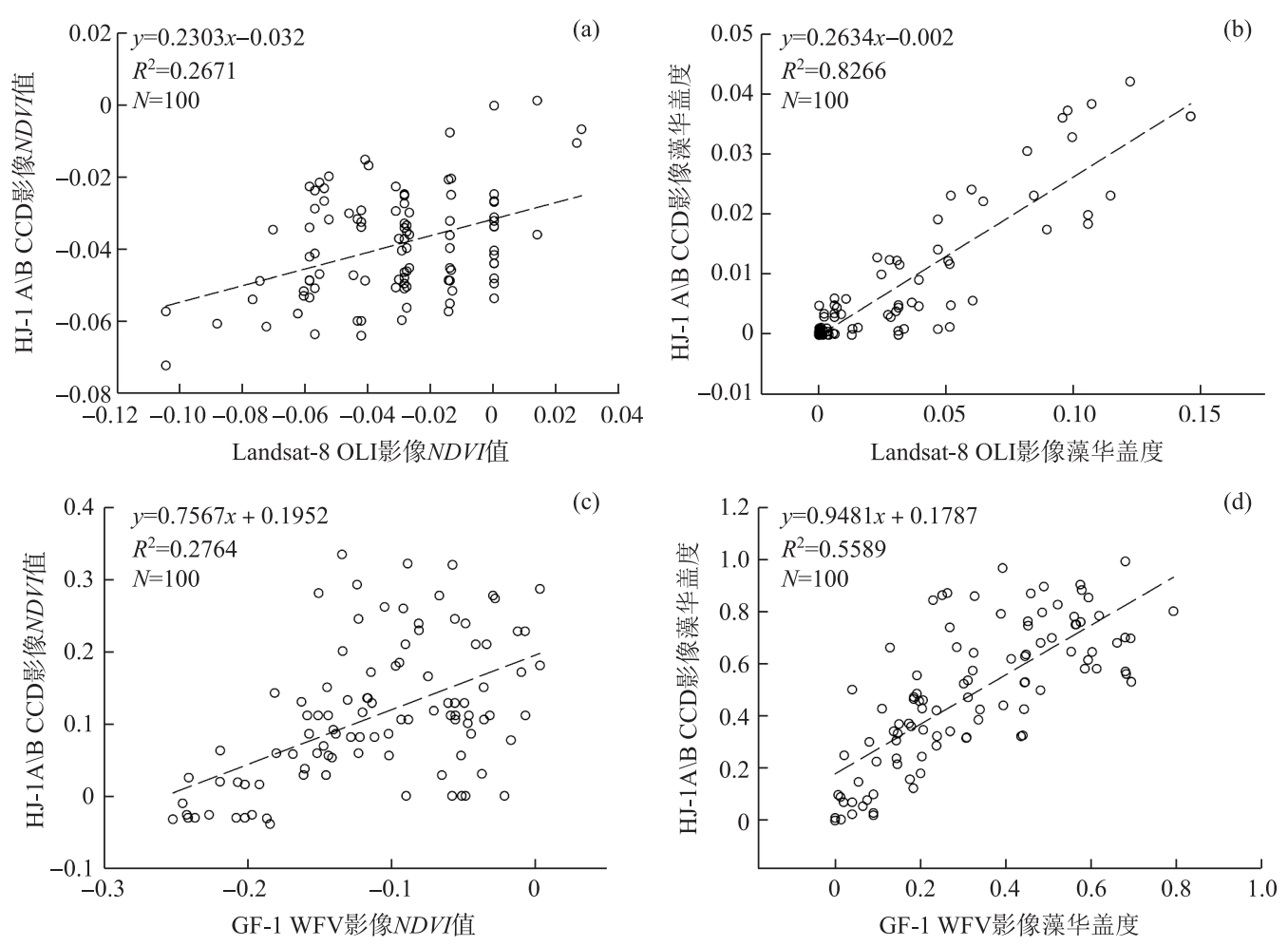

图 $11 \mathrm{HJ}-1 \mathrm{~A} \backslash \mathrm{B}$ 与 Landsat-8、GF-1 NDVI 值关系 $(\mathrm{a}, \mathrm{c}) 、$ 藻华盖度关系 $(\mathrm{b}, \mathrm{d})$

Fig.11 HJ-1A $\backslash$ B, Landsat-8 NDVI value relationship(a,c), algal canopy degree(b,d)

从 2016 年月际间柱状图比较 (图 13),排除冬季水库水面结冰,4-6 月水质状况较好, 从 7-9 月开始 于桥水库水质呈下降趋势, 蓝藻水华暴发加剧, 面积增大.

\section{4 结论与展望}

针对内陆小型水域于桥水库, 分析了 HJ-1A \B、GF-1 和 Landsat-8 高分辨率卫星对小型水库蓝藻水华的 联合监测能力; 同时根据各卫星波段设置特点, 选取 $N D V I$ 指数, 构建了蓝藻水华提取算法, 获取了于桥水库 蓝藻水华的面积和空间分布情况, 最后利用尺度转换重采样的方式保持不同卫星观测结果的一致性. 该方 法较精确地获得于桥水库蓝藻水华暴发面积和分布状况. 相比单一数据源, 该方法联合 HJ-1A $\backslash B 、 G F-1$ 和 Landsat-8 卫星监测水华,对于统计水华发生频次、水华累计发生时间及确定单次水华持续时间等更加准确 


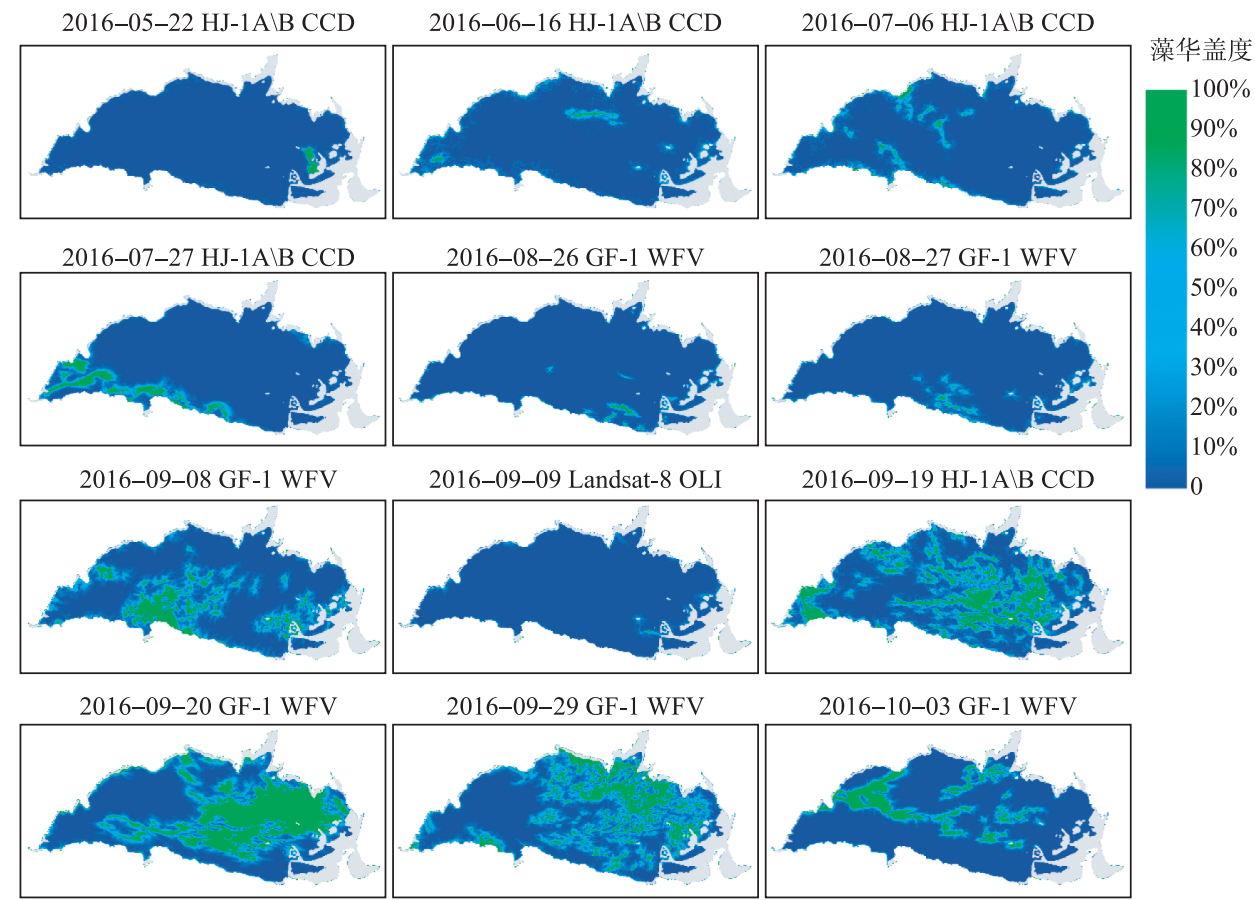

图 12 高分辨率卫星联合监测 2016 年藻华最大暴发面积对比

Fig. 12 Comparison of the largest outbreak area of algal blooms in 2016 by high-resolution satellite observations

客观. 因此,针对小型水体,采用多星联合观测的方法是 目前业务化运行最为合适的选择. 另外, 从该方法提取的 结果来看, 2016 年于桥水库蓝藻水华暴发较为频繁, 尤 其是 $9 、 10$ 月水质持续恶化, 问题较为突出.

伴随着卫星传感器和反演算法的不断增加和发展, 卫星遥感技术已经在大型湖泊水华监测中得到广泛应 用; 但小型水体面积较小, 水体光学特性复杂, 受人类活 动扰动强度较大. 建议综合利用各种传感器的优点, 特别 是高分辨率卫星的优势, 加强多源卫星的联合观测和数 据一致性评价,形成适用于小型水体的高分卫星立体监 测体系和标准化水华监测产品, 为形成适用于不同类型 水体的蓝藻水华监测提供解决方案.

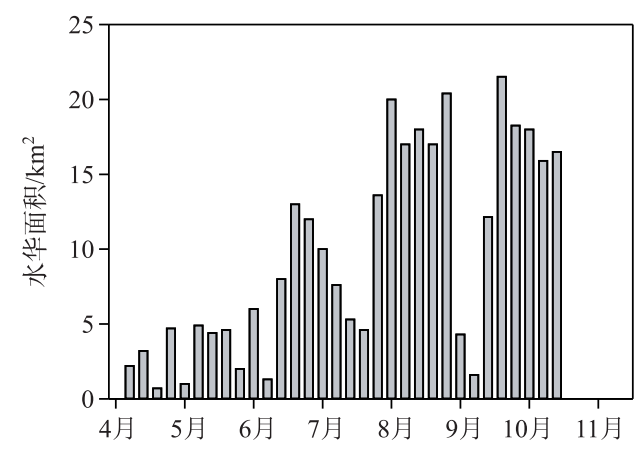

图 13 月际间蓝藻水华面积比较

Fig.13 Comparison of monthly area of algae blooms in cyanobacteria

\section{5 参考文献}

[ 1 ] Han BP. Reservoir ecology and limnology in China: A retrospective comment. J Lake Sci, 2010, 22(2) : 151-160. DOI: 10.18307/2010.0201. [ 韩博平. 中国水库生态学研究的回顾与展望. 湖泊科学, 2010, 22(2): 151-160.]

[ 2 ] Zhao MX, Han BP. Analysis of factors affecting cyanobacteria bloom in a tropical reservoir( Tangxi Reservoir, China). Acta Ecologica Sinica, 2005, 25(7): 1554-1561. [ 赵孟绪, 韩博平. 汤溪水库蓝藻水华发生的影响因子分析. 生态学 报, 2005, 25(7): 1554-1561.]

[ 3 ] Cui GB, Liu L, Yao Q et al eds. Research on the mechanism of eutrophication control in Taihu Basin. Beijing: China Water \& Power Press, 2009. [崔广柏, 刘凌, 姚琪等. 太湖流域富营养化控制机理研究. 北京: 中国水利水电出版 社, 2009.] 
[ 4 ] Xie P ed. Reading about the histories of cyanobacteria, eutrophication and geological evolution in Lake Chaohu. Beijing: Science Press, 2009. [谢平. 翻阅巢湖的历史: 蓝藻、富营养化及地质演化. 北京: 科学出版社, 2009.]

[ 5 ] Kong FX, Ma RH, Gao JF et al. The theory and practice of prevention, forecast and warning on cyanobacteria bloom in Lake Taihu. J Lake Sci, 2009, 21(3) : 314-328. DOI: 10.18307/2009.0302. [ 孔繁翔, 马荣华, 高俊峰等. 太湖蓝藻 水华的预防、预测和预警的理论与实践. 湖泊科学, 2009, 21(3):314-328.]

[ 6 ] Duan HT, Zhang SX, Zhang YZ. Cyanobacteria bloom monitoring with remote sensing in Lake Taihu. J Lake Sci, 2008, 20(2) : 145-152. DOI: 10.18307/2008.0202. [ 段洪涛, 张寿选, 张洲智. 太湖蓝藻水华遥感监测方法. 湖泊科学, $2008,20(2): 145-152$.

[ 7 ] Li XW, Niu ZC, Jiang S et al. Design of intensity index and build-up degree classification algorithm development for cyanobacterica blooms in Lake Taihu based on satellite remote sensing. Administration and Technique, 2011, 23(5) : 23-30. [李旭文, 牛志春, 姜晟等. 基于卫星影像的太湖蓝藻水华遥感强度指数和等级划分算法设计. 环境监测管理与技 术, 2011, 23(5) : 23-30.]

[ 8 ] Hu CM. A novel ocean color index to detect floating algae in the global oceans. Remote Sensing of Environment, 2009, 113: 2118-2129.

[ 9 ] Hu CM, Lee ZP, Ma RH et al. Moderate Resolution Imaging Spectroradiometer (MODIS) observations of cyanobacteria blooms in Taihu Lake, China. Journal of Geophysical Research, 2010, 115: 4002-4020.

[10] Zhang YC, Ma RH, Duan HT et al. A novel algorithm to estimate algal bloom coverage to subpixel resolution in Lake Taihu. IEEE Journal of Selected Topics in Applied Earth Observations and Remote Sensing, 2014, 7 (7) : 3060-3068.

[11] Li XJ, Ma ZZ, Zhou ZY. Scale analysis of cyanobacteria bloom from MODIS and HJ-1A/1B observations. Remote Sensing Information, 2016, 31(5) : 108-113. [李晓俊, 马泽忠, 周志跃. 一种蓝藻水华提取空间尺度效应分析. 遥感信息, 2016, 31(5): 108-113.]

[12] Zhou XS, Ji YL, Kong FQ et al. Primary study on the stratification characteristics of Yuqiao Reservoir based on site monitoring parameters of water quality. Haihe Water Resources, 2015, (2): 19-21. [周绪申, 计亚丽, 孔凡青等. 基于水质 现场监测参数的于桥水库水体分层特征初步研究. 海河水利, 2015, (2) : 19-21.]

[13] Chang C, Feng P, Sun DM et al. Prediction of the alga growth in a reservoir based on the stepwise cluster analysis. China Environmental Science, 2015, 35(9): 2805-2812. [ 常淳, 冯平, 孙冬梅等. 基于逐步聚类分析的水库浮游藻类生长 预测. 中国环境科学, 2015, 35(9) : 2805-2812.]

[14] Li XJ, Sun XY, Kong QJ. Investigation and analysis of water pollution factors in Yuqiao Reservoir. Haihe Water Resources, 2013, (6) : 27-28. [李雪娇, 孙翔宇, 孔庆杰. 于桥水库水污染因素调查分析. 海河水利, 2013, (6): 27-28.]

[15] Xing HY, Bao Z, Ning WH. Water quality status appraisal and protection countermeasures for water sources in Panjiakou and Daheiting Reservoirs. Haihe Water Resources, 2009, 24(4) : 24-27. [刑海燕, 暴柱, 宁文辉. 潘家口、大黑汀水库 水源地水质现状评价与保护对策. 海河水利, 2009, 24(4): 24-27.]

[16] Ma RH, Kong FX, Duan HT et al. Spatio-temporal distribution of cyanobacteria blooms based on satellite imageries in Lake Taihu, China. J Lake Sci, 2008, 20(6) : 687-694. DOI: 10.18307/2008.0605. [ 马荣华, 孔繁翔, 段洪涛等. 基 于卫星遥感的太湖蓝藻水华时空分布规律认识. 湖泊科学, 2008, 20(6) : 687-694.]

[17] Zhang J, Chen LQ, Chen XL et al. Evaluation of monitoring ability of cyanobacterial blooms by HJ-1B and Landsat: A case study of Erhai Lake. Journal of Water Resources and Water Engineering, 2016, 27(4):38-43. [张娇, 陈莉琼, 陈 晓玲等. HJ-1B 和 Landsat 卫星蓝藻水华监测能力评估. 水资源与水工程学报, 2016, 27 (4) : 38-43.]

[18] Xing Q, Hu C. Mapping macroalgal blooms in the Yellow Sea and East China Sea using HJ-1 and Landsat data: Application of a virtual baseline reflectance height technique. Remote Sensing of Environment, 2016, 178: 113-126.

[19] Nai ZJ, Duan HT, Zhu L et al. A novel algorithm to monitor cyanobacterial blooms in Lake Taihu from HJ-CCD imagery. $J$ Lake Sci, 2016, 28(3) : 624-634. DOI: 10.18307/2016.0319. [佴兆骏, 段洪涛, 朱利等. 基于环境卫星 CCD 数据的 太湖蓝藻水华监测算法研究. 湖泊科学, 2016, 28(3): 624-634.]

[20] Zhang J, Chen LQ, Chen XL. Monitoring the cyanobacterial blooms based on remote sensing in Lake Erhai by FAI. J Lake $S c i, 2016,28(4)$ : 718-725. DOI: 10.18307/2016.0404. [张娇, 陈莉琼, 陈晓玲. 基于 FAI 方法的洱海蓝藻水华遥 感监测. 湖泊科学, 2016, 28(4) : 718-725.]

[21] Wu JG ed. Landscape ecology pattern, process, scale and hierarchy. Beijing: Higher Education Press, 2000: 11-12. [乌 建国. 景观生态学: 格局、过程、尺度与等级. 北京: 高等教育出版社, 2000: 11-12.] 